\title{
IR Divergences in Inflation and Entropy Perturbations
}

\author{
Wei Xue ${ }^{1 \mathrm{a}}$, Xian $\mathrm{Gao}^{2,3,4 \mathrm{~b}}$ and Robert Brandenberger ${ }^{1 \mathrm{c}}$ \\ 1) Department of Physics, McGill University, \\ 3600 Rue Université, Montréal, Québec, Canada H3A $2 T 8$ \\ 2) Astroparticule 83 Cosmologie, Université Denis Diderot-Paris 7, \\ 10 rue Alice Domon et Léonie Duquet, 75205 Paris, France \\ 3) $\mathcal{G} \mathbb{R} \varepsilon \mathbb{C O}$, Institut d'Astrophysique de Paris, \\ Université Pierre et Marie Curie-Paris 6, \\ 98bis Boulevard Arago, 75014 Paris, France and \\ 4) Laboratoire de Physique Théorique, École Normale Supérieure, 24 rue Lhomond, 75231 Paris, France
}

\begin{abstract}
We study leading order perturbative corrections to the two point correlation function of the scalar field describing the curvature perturbation in a slow-roll inflationary background, paying particular attention to the contribution of entropy mode loops. We find that the infrared divergences are worse than in pure de Sitter space: they are power law rather than logarithmic. The validity of perturbation theory and thus of the effective field theory of cosmological perturbations leads to stringent constraints on the coupling constants describing the interactions, in our model the quartic self-interaction coupling constant of the entropy field. If the self coupling constant is larger than some critical value which depends in particular on the duration of the inflationary phase, then perturbation theory breaks down. Our analysis may have implications for the stability of de Sitter space: the quantum effects which lead to an instability of de Sitter space will be larger in magnitude in the presence of entropy fluctuations.
\end{abstract}

\footnotetext{
a xuewei@hep.physics.mcgill.ca

bxgao@apc.univ-paris7.fr

c rhb@physics.mcgill.ca
} 


\section{INTRODUCTION}

The inflationary universe scenario provides an explanation for the observed inhomogeneities in the distribution of galaxies and the anisotropies of the cosmic microwave background. According to inflationary cosmology, the primordial fluctuations arise from initial vacuum perturbations of the canonical variable which describes the coupled metric and matter inhomogeneities ( see [1] for a overview of cosmological perturbation theory). The standard computations are done at tree level. However, since Einstein's gravitational theory is non-linear, it is not valid to neglect interactions which appear at higher order in perturbation theory. Such interactions will lead to non-Gaussianities in the spectrum of cosmological perturbations, a topic which has received a lot of recent interest (see e.g. [2] for an early reference). Interactions also effect the two point functions via loop corrections during inflation (see e.g. [3], and they can even lead to back-reaction effects on the cosmological background itself, see e.g. [4,5] for early works computing the effects of graviton and scalar loops, respectively).

In order to study quantum field theory during inflation or, more ambitiously, to understand quantum gravity, it is necessary to systematically treat perturbation theory. As a nonrenormaliziable theory, General Relativity needs an infinite number of counter-terms to get rid of the ultraviolet (UV) divergences (see e.g. the textbook of [6] and also the more recent articles $[3,7])$. Since in inflationary cosmology it is assumed that UV modes originate in their vacuum state, the contribution of UV physics to loop corrections of correlation functions is tiny and beyond the interest for observations. On the other hand, the contributions of infrared (IR) modes to correlation functions can be large in inflationary cosmology due to the large phase space of such IR modes. It is the Hubble radius $H^{-1}$ which separates UV from IR scales. The Hubble radius is approximately constant (in physical coordinates) during the phase of inflationary expansion. For a massless field there are both UV and IR divergences. If we employ a brute cutoff renormalization scheme to deal with the two kinds of divergences, then the UV cutoff scale must be taken to be fixed in physical coordinates, while the IR cutoff is a comoving one [8]. Hence, during a period of accelerated expansion of space the phase space of IR modes increases. Thus, in contrast to UV physics, IR physics may well be lead to observationally relevant effects (see e.g. $[4,5,9,10]$ since the long distance modes can back-react on the semi-classical space-time or on other gravitational perturbation modes. There have been a lot of studies on the effects of infrared divergences on inflationary observables (see [11] for a recent review and e.g. [12] for some original studies). There is an emerging consensus that in the presence of only adiabatic fluctuations, the effect on cosmo- 
logical observables will be small [10, 13]. However, as stressed in [14] in the context of studies of the effect of back-reaction of the infrared modes on the background cosmology itself, the story changes completely if entropy modes are present. Since matter contains many fields, it is not realistic to neglect the presence of these entropy modes.

There is a second aspect of IR problems in cosmology: As pointed out in [17], perturbation theory may break down because of the contribution of IR modes to loop integrals. In thermal field theory this is called the "Linde problem": In the thermal ensemble the soft gluons contributing as IR loops to Feynman diagrams cause the complete failure of perturbation theory since in fact the transverse gluons obtain a mass via screening. In de Sitter space-time, a scalar field with a mass which is tiny compared with the Hubble scale will cause the same problem as in thermal field theory (see e.g. [15] and also [16]). For an exactly massless field, the IR loops will be logarithmically divergent ${ }^{1}$.

The analysis of infrared divergences in inflationary cosmology is complicated by the fact that we must treat both metric and matter fluctuations since they are coupled via the Einstein equations. This mixing must be taken into account in the computation of observables. The observable of particular interest is the curvature fluctuation variable $\zeta[1,19]$. In particular, in the case of a massive matter field in the case of inflationary cosmology it turns out that the tilt of the curvature perturbations and of the gravitational wave spectrum is similar to what is obtained in the case of a massive scalar field in pure de Sitter space-time - thus there is a power law IR divergence in inflationary space-time.

In this paper, we analyse the IR divergences arising via loop effects in the two point correlation function of the curvature fluctuation variable $\zeta$ in the case of an inflationary space-time. We find a power law divergence rather than the logarithmic divergence which appears in pure de Sitter space-time. The IR divergences can be regulated, but the regulator is related to the initial condition at the beginning of the phase of inflationary expansion. Field theory in an expanding space-time is different from quantum field theory in Minkowski space-time: As the inflationary phase begins, the vacuum keeps generating UV modes deep inside the Hubble radius, modes which exit the Hubble radius, freeze out and are subsequently squeezed and become the IR ensemble of quasi-classical fluctuations which will back-react on the background $[4,5]$ and impact the newlygenerated gravitational modes. Compared with the mild logarithmic divergence of pure de Sitter space-time, the power law IR divergences in inflationary cosmology will put stronger constraints

\footnotetext{
${ }^{1}$ See also [18] for an independent study of how infrared divergences in inflationary cosmology can lead to a breakdown of cosmological perturbation theory.
} 
on the applicability or perturbative methods in inflation.

However, it has been shown that the leading order contributions of IR divergences in inflationary cosmology cancel, which can be proven by applying either the in-in formalism [11] or by semiclassical relations [9]. Also, in the case of pure adiabatic fluctuations, IR divergences from naive curvature perturbations can be locally gauged away (see e.g. [20-23]), while in the presence of entropy perturbations the IR effects are locally measurable and can no longer be gauged away. Thus, the divergences from entropy modes are physically relevant [14]. Meanwhile, the entropy perturbations are the source of curvature perturbations and, more specifically, lead to the growth of curvature perturbations on super-Hubble scales. Thus, IR divergences will effect the observed curvature fluctuations. Hence, the fact that the curvature fluctuations are time-dependent, the presence of entropy perturbations, and the stronger IR divergences from loop effects will change the story of IR divergences in inflationary cosmology compared to what happens in the case of a spectator scalar field in de Sitter space.

In this paper, we will study the power law IR divergences and the entropy perturbations in inflationary cosmology. In Sec. II, we will discuss the power law divergences arising in single field inflation models. This effect is general, and can be applied to multiple field models as well. The new feature in multi-field models is the presence of entropy fluctuations which we study in the subsequent section, We first consider massless entropy modes, and then massive ones. We derive non-trivial bounds on the magnitude of the self-coupling of the entropy modes which have to be satisfied in order for perturbation theory to remain applicable. Finally, we study the case of scalar matter fields which are not coupled to the inflaton. However, there is a coupling with gravity, it this also results in constraints on the self coupling constant if perturbation theory is to be reliable.

\section{CASE OF CONSERVED $\zeta$}

In this section, we compute the leading loop corrections to the two point function of the curvature fluctuation $\zeta$ for single field inflation, in which case $\zeta$ is conserved on super-Hubble scales. As we will show a bit later, the most convenient gauge for the loop calculations is comoving gauge. We take into account that the Hubble parameter is not exactly constant during inflation. We show that this has an essential effect on IR divergences. Our discussion is based on describing space-time using Einstein's gravitational action, and considering matter to be composed of a set of minimally coupled scalar fields. 


\section{II.1. Single Field Inflation}

Single field inflation is obtained by using the condensation of a single scalar field $\phi$ with a nontrivial but very flat potential in order to drive space to expand nearly exponentially. The resulting space-time background is often called "(quasi)-de Sitter space", The background metric can be written in the form

$$
\mathrm{d} s^{2}=-\mathrm{d} t^{2}+a^{2}(t) \mathrm{d} x^{2}=a^{2}(\eta)\left(-\mathrm{d} \eta^{2}+\mathrm{d} x^{2}\right)
$$

where $t$ is physical time, $x$ are the comoving spatial coordinates, $\eta$ is the conformal time, and the scale factor $a(t)$ takes the form $a(t)=e^{H t}=-\frac{1}{H \eta}$ in the case of pure de-Sitter space.

When introducing gravitational fluctuations, it is important to keep in mind that gravity is similar to a gauge field theory in that there is a redundant local symmetry, namely space-time diffeomorphism invariance. Out of the ten naive metric degrees of freedom, there are only six physical ones - two scalar, two vector and two tensor (see [1] for an in-depth discussion). The two tensor modes are the two polarization states of the gravitons, in an expanding universe the two vector modes can be neglected, and the two scalar metric and one matter degrees of freedom are constrained by two of the Einstein equations, leaving one physical mode. To get rid of the redundancy in the scalar sector, we can choose different gauges, but physics will not depend on our gauge choices. Uniform spatial curvature gauge is obtained by choosing the time slices such that the scalar metric perturbation (the component of the metric corresponding to the scalar mode) vanishes. In this case the scalar matter field perturbation will be non-vanishing. The spatial part of the metric takes the form

$$
g_{i j}=a^{2}\left(\delta_{i j}+h_{i j}\right)
$$

where $h_{i j}$ is a transverse and traceless tensor representing the gravitons.

The other convenient gauge in the case of single field inflation is the comoving gauge where the matter field fluctuation $\delta \phi$ is set to zero and the perturbation appears as a fluctuation of the spatial curvature:

$$
\begin{aligned}
\delta \phi & =0 \\
g_{i j} & =a^{2}\left[(1+2 \zeta) \delta_{i j}+h_{i j}\right] .
\end{aligned}
$$

The remaining degrees of freedom of the perturbations in the specific time slice are curvature perturbation $\zeta$ and graviton $h_{i j}$. As we will argue below, for our purposes the comoving gauge has advantages. 
At quadratic order, scalar metric fluctuations and gravitons decouple. In the comoving gauge, the quadratic actions of curvature perturbations and gravitons are as follows

$$
S_{2, s}=\frac{1}{2} \int \mathrm{d} t \mathrm{~d} x^{3} \epsilon\left[a^{3} \dot{\zeta}^{2}-a(\partial \zeta)^{2}\right]
$$

and

$$
S_{2, t}=\frac{1}{8} \int \mathrm{d} t \mathrm{~d} x^{3}\left[a^{3} \dot{h}_{i j}^{2}-a\left(\partial_{l} h_{i j}\right)^{2}\right]
$$

where the subscripts $s$ and $t$ stand for the scalar and tensor contributions, respectively. In the above, we are working in Planck units, i.e. to obtain the dimensionless metric components of (3), we must divide the values of $\zeta$ and $h$ appearing in the above action by the Planck mass $m_{p l}$.

At quadratic order, each Fourier mode evolves independently. The solutions of the equations of motion for $k$ modes normalized to correspond to quantum vacuum perturbations on sub-Hubble scales are

$$
\zeta_{k}=\frac{H}{\sqrt{2 \epsilon} \sqrt{2 k^{3}}}(1+i k \eta) e^{-i k \eta}
$$

and

$$
h_{ \pm, k}=\frac{H}{\sqrt{k^{3}}}(1+i k \eta) e^{-i k \eta} .
$$

Here $\epsilon$ is the first slow-roll parameter, which is defined as the rate of change of the Hubble scale in one Hubble time:

$$
\epsilon=-\frac{\dot{H}}{H^{2}}=\frac{\dot{\phi}^{2}}{2 H^{2}}
$$

In de Sitter space, the Hubble scale is constant, while during inflation, the Hubble scale is decreasing since the inflaton is slowly rolling down its potential. The first slow roll parameter is required to be very small during the inflationary phase, and moreover, must be kept small for the duration of inflation which corresponds to at least 60 e-foldings. Hence there are other parameters which characterize the rate of change of $\epsilon$, and they are also required to be small. The second slow-roll parameter is

$$
\eta \equiv \frac{V^{\prime \prime}}{V} \sim-\frac{\ddot{\phi}}{H \dot{\phi}}+\frac{1}{2} \frac{\dot{\phi}^{2}}{H^{2}}=2 \epsilon-\frac{\dot{\epsilon}}{H \epsilon}
$$

Sometimes we require inflation to last more than 60 e-foldings, such that $\epsilon$ is slowly changing with $\frac{\dot{\epsilon}}{H \epsilon} \sim \mathcal{O}\left(\epsilon^{2}\right)$ which is of next order of $\epsilon$. In this case $\eta \simeq 2 \epsilon$. 
Although the Hubble scale is not exactly constant, $\zeta$ and $h$ are good quantities to describe the perturbations in inflationary cosmology since they are conserved outside the Hubble radius as long as there are no entropy perturbations. The reason that they are conserved is the rescaling symmetry of the equations, as can be seen from the action which is total derivative outside the horizon, such that the curvature and graviton perturbations are not dynamical at all after Hubble radius crossing.

Therefore, the amplitudes of $\zeta_{k}$ and $h_{k}$ for modes whose wavelengths are larger than the Hubble radius, can be described by the Hubble scale and the slow roll parameters at the time when the modes cross the Hubble radius:

$$
\zeta_{k}=\frac{H_{*}}{\sqrt{2 \epsilon_{*}} \sqrt{2 k^{3}}}(1+i k \eta) e^{-i k \eta}
$$

and

$$
h_{ \pm, k}=\frac{H_{*}}{\sqrt{k^{3}}}(1+i k \eta) e^{-i k \eta},
$$

where subscripts $*$ mean that the corresponding quantities are evaluated at the Hubble crossing time. Although on super-Hubble scales curvature perturbations and gravitons do not depend on time, they are functions of $k$ because inflation is not exactly de Sitter.

$$
\begin{aligned}
\left\langle\zeta_{k} \zeta_{k}\right\rangle & \simeq \frac{H^{4}}{2 k^{3} \dot{\phi}^{2}} \\
\left\langle h_{k} h_{k}\right\rangle & \simeq \frac{H^{2}}{k^{3}} .
\end{aligned}
$$

The $k$ dependence of the perturbations is characterized by spectral indices $n_{s}$ and $n_{t}$, both of which are small numbers:

$$
n_{s}-1 \equiv k \frac{\mathrm{d}}{\mathrm{d} k} \log \left(\frac{H^{4}}{\dot{\phi}^{2}}\right)=\frac{\mathrm{d}}{H \mathrm{~d} t_{*}} \log \left(\frac{H^{4}}{\dot{\phi}^{2}}\right)=2(\eta-3 \epsilon),
$$

and

$$
n_{t} \equiv k \frac{\mathrm{d}}{\mathrm{d} k} \log \left(H^{2}\right)=-2 \epsilon
$$

In inflationary models, the gravitational waves have a red spectrum, which means $n_{t}<0$. A blue spectrum is only possible if the Null Energy Condition for matter is violated or some non-standard sound speed is introduced ${ }^{2}$. Cosmic microwave (CMB) anisotropy experiments can measure $n_{s}$, and current data indicate that $n_{s}-1$ is slightly negative.

\footnotetext{
${ }^{2}$ The fact that inflation leads to a red tilt of the tensor power spectrum leads to a way to falsify inflation. There are other cosmological scenarios such as "string gas cosmology" [24, 25] which predicts a blue spectrum of gravitational waves $[26]$
} 


\section{II.2. Gauges}

In this subsection, we compare different gauges and the resulting different forms of the scalar perturbations during inflation, and we suggest that the comoving gauge is the best gauge to compute IR loops. Observables are gauge-invariant and the physics does not depend on the gauge choice. However, in terms of being able to reliably compute the observables, there often exists a choice of gauge which is particularly convenient.

Two other gauges besides the comoving gauge have often been used in recent loop analyses of inflation. The first is a gauge which arises in the effective field theory of inflation in which one uses a Goldstone modes of gravity perturbations (see e.g. $\pi$ [27]). However, for dealing with IR loops this gauge has problems. The main idea of $\pi$ effective field theory is to apply the Goldstone equivalence principle to the inflationary perturbation theory. At high energy, the mixing between Goldstone mode and metric perturbation modes is not important, and then the scalar perturbation can be treated as a Goldstone boson $\pi$. For single field inflation models, this effective field theory breaks down for modes whose energy corresponds to $E<\epsilon^{1 / 2} H, \pi$. But a large contribution to IR loops comes from modes in this range.

A second frequently used gauge is the uniform density gauge $\zeta=0$. In this case, the fluctuations are carried by the scalar field perturbation $\delta \phi$ which is non-zero. It is well known that $\delta \phi$ is not conserved outside horizon. To calculate the tree level two point function of curvature perturbation, it is convenient to make use of the fact that $\zeta$ is conserved after crossing the Hubble horizon. Thus, it can be calculated at the Hubble crossing time. With the relationship matter fluctuations $\delta \phi$ and metric fluctuations $\zeta$ at this time (valid in any gauge), we can obtain the curvature perturbations while working in the uniform density gauge. However, in this gauge the IR loop calculation needs to take into account the time evolution of the integrands on super-Hubble scales, and thus involves an integration over the whole time of inflation. In other words, if we use $\delta \phi$, we must take into account the time dependence of $\delta \phi$ after Hubble crossing time.

On the other hand, working in comoving gauge in the single field model of inflation, the curvature perturbation $\zeta$ is conserved outside of the Hubble radius. The time dependence of correlation functions of the fluctuation variables in this gauge is trivial. When we perform the time integration in loop integrals, the IR modes of $\zeta$ are time-independent and can be taken out of the time integral. Thus, the computations are technically easier. Note, however, that the small tilt from the k-dependence of $\zeta$ is essential for the IR loop diagram integration. In the UV, the tilt of the spectrum is not crucial, since deep inside the Hubble radius the WKB method can be used [28]. 
To summarize, we conclude that the comoving gauge is the best one for computing IR loops in single field inflation models. In multi-field models of inflation and in the presence of non-vanishing entropy perturbations, $\zeta$ is no longer conserved. The IR divergences of inflation becomes more interesting as we will discuss it in the next section.

\section{II.3. Interactions}

After choosing the gauge, the next step is to gather the interaction terms for loop calculation. In comoving gauge, there is no interactive ghost field and thus we need not consider any ghost terms. In single field inflation with sound speed $c_{s}=1$, we use $\epsilon$ as second expansion parameter for the gravitational action. The first expansion parameter is the amplitude of the linear fluctuation $\zeta$, and for each order in $\zeta$ we can expand the corresponding terms in the interaction Lagrangian in terms of $\epsilon$. Here we just keep the leading terms in $\epsilon$ for every kind of interaction. In addition,

there are terms proportional to $\frac{\delta \mathcal{L}}{\delta \zeta}$ which can be dealt with by redefinition of $\zeta$ or $h_{i j}$. These terms are neglected here because they have nothing to do with the leading IR divergences.

In this section, we will list all interaction terms that are relevant to one loop corrections to two-point function, and we also write down the higher interaction terms since they can be used to estimate the order of magnitude of the two loop corrections to two-point functions, or the loop corrections to $n$-point correlation function. In the second part of the section, we will give the method to estimate the result of the loop corrections. In the following, we will use conformal time because it is convenient in order to estimate the order of the loop corrections.

The second order action is $\mathcal{O}(\epsilon)$ and takes the form $[1,29,30]$

$$
S_{2}=\int \mathrm{d} \eta \mathrm{d} x^{3} \epsilon a^{2}\left(\zeta^{\prime 2}-(\partial \zeta)^{2}\right)
$$

The cubic interaction term was first calculated in [31]. It is $\mathcal{O}\left(\epsilon^{2}\right)$ and is given by

$$
S_{3}=\int \mathrm{d} \eta \mathrm{d} x^{3} \epsilon^{2} a^{2}\left[\zeta^{\prime 2} \zeta-(\partial \zeta)^{2} \zeta-2 \zeta^{\prime} \partial_{i} \chi \partial_{i} \zeta\right]
$$

where

$$
\partial^{2} \chi=\zeta^{\prime}
$$

The interaction Hamiltonian is written as

$$
H_{3}^{(I)}=-\mathcal{L}_{3} .
$$


The leading fourth-order term is of the same order as the cubic interaction $\mathcal{O}\left(\epsilon^{2}\right)$ (it was first computed in [32])

$$
S_{4}=\int \mathrm{d} t \mathrm{~d} x^{3} \epsilon^{2} a^{2}\left\{-\beta_{j} \partial^{2} \beta_{j}-\frac{1}{a H} \partial^{-2} \Sigma\left[\zeta^{\prime 2}+(\partial \zeta)^{2}\right]-3\left(\partial^{-2} \Sigma\right)^{2}-4 \zeta^{\prime} \beta_{j} \partial_{j} \zeta\right\}
$$

where

$$
\frac{1}{2} \partial^{4} \beta_{i} \equiv \delta^{r s}\left(\partial_{i} \Sigma_{r s}-\partial_{(r} \Sigma_{s) i}\right)
$$

and

$$
\Sigma_{r s} \equiv \partial_{r} \zeta^{\prime} \partial_{s} \zeta+\zeta^{\prime} \partial_{r s} \zeta
$$

We have also used $\Sigma \equiv \delta^{r s} \Sigma_{r s}$. If keep the leading order, the interaction Hamiltonian is also the minus Lagrangian,

$$
H_{4}^{(I)}=-\mathcal{L}_{4}
$$

The fifth order and sixth order terms are more and more complicated, and we just mention the order in $\epsilon$ of the leading terms in these interaction terms, and we omit partial derivatives on $\zeta$ in the expressions (this expansion was first discussed in [33])

$$
S_{5} \sim \int \mathrm{d} t \mathrm{~d} x^{3} a \epsilon^{3} \zeta^{5}
$$

and

$$
S_{6} \sim \int \mathrm{d} t \mathrm{~d} x^{3} a \epsilon^{3} \zeta^{6}
$$

For the loop corrections, $S_{3}$ is subdominant compared to $S_{4}$. The one loop diagram with $S_{3}$ interactions has one more vertex than the one with $S_{4}$. It is thus suppressed by $\epsilon^{2}$, but it has one more propagator, which gives an enhancement of $1 / \epsilon$. Therefore, $S_{3}$ is subdominant.

To compute loop diagrams we must identify the vertex factors and propagators. Concerning the vertex factors,

$$
S_{4} \quad \text { vertex } \mapsto \frac{\epsilon^{2}}{H^{2}}
$$

and

$$
S_{3} \quad \text { vertex } \mapsto \frac{\epsilon^{2}}{H^{2}}
$$


Every internal and external curvature $\zeta$ propagator gives

$$
\zeta \text { propagator } \mapsto \frac{H^{2}}{\epsilon},
$$

and the graviton propagator (which will be relevant since there are interactions between scalar metric fluctuations and gravitons at higher than quadratic order) is

$$
h \text { propagator } \mapsto H^{2},
$$

which comes from the action for free gravitons which is

$$
S_{h 2}=\frac{1}{8} \int \mathrm{d} \eta \mathrm{d} x^{3} a^{2}\left(h_{i j}^{\prime} h_{i j}^{\prime}-\partial_{l} h_{i j} \partial_{l} h_{i j}\right)
$$

Now let us consider the individual interaction terms. The cubic interaction term between two scalars and a graviton is

$$
S_{\zeta 2 h}=\int \mathrm{d} \eta \mathrm{d} x^{3} \epsilon a^{2} h_{i j} \partial_{i} \zeta \partial_{j} \zeta
$$

The cubic interaction term between one scalar and two graviton is subdominant in $\epsilon$ since it has the form

$$
S_{\zeta h 2}=\int \mathrm{d} \eta \mathrm{d} x^{3} \frac{\epsilon}{2} H a^{3} h_{i j}^{\prime} h_{i j}^{\prime} \partial^{-2} \zeta^{\prime}
$$

The quartic interaction term between two scalars and two gravitons is

$$
S_{\zeta 2 h 2}=\int \mathrm{d} \eta \mathrm{d} x^{3} \epsilon a^{2}\left(-\frac{1}{2} h_{i l} h_{l j} \partial_{i} \zeta \partial_{j} \zeta\right)
$$

Counting the order of $\epsilon$ is a easy way to know whether a particular interaction is dominant or not. Let us consider the leading contributions to the two point function of $\zeta$ :
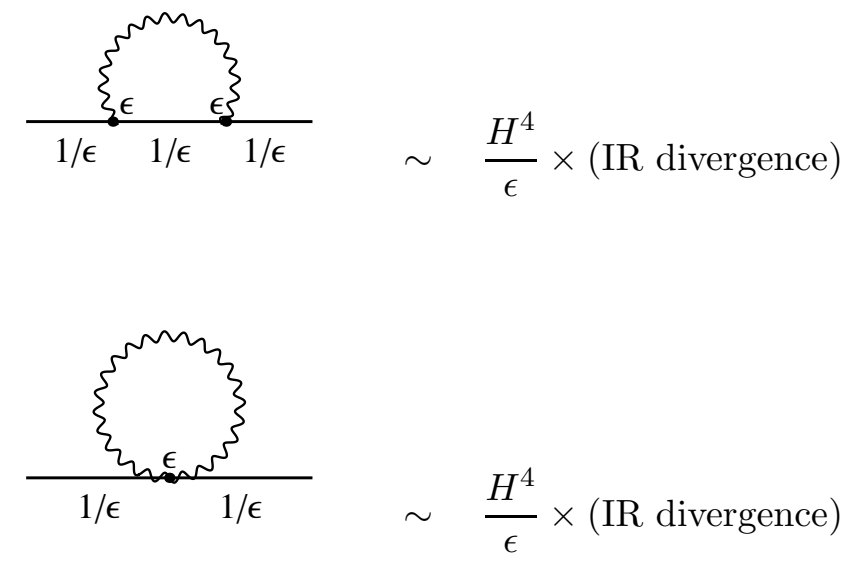

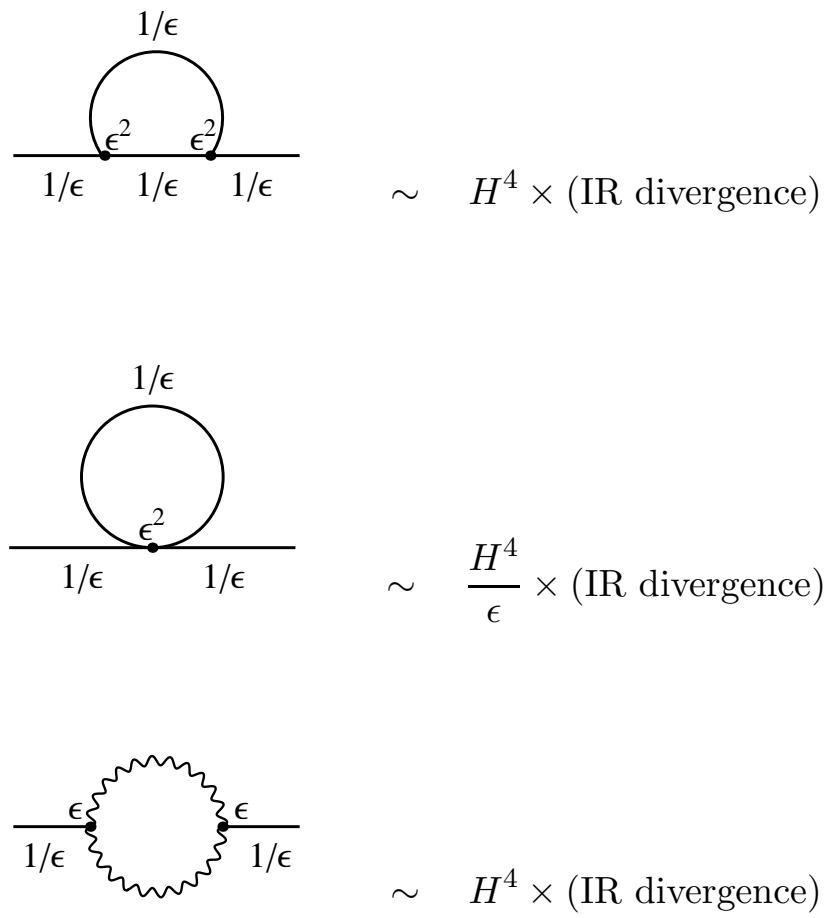

3 where the wavy lines stand for gravitons and the solid lines are curvature propagators. The dominant interaction terms at one loop are $S_{4}, S_{\zeta 2 h}$ and $S_{\zeta 2 h 2}$. This counting method can be easily generalized to $n$-point correlation functions and to higher loop corrections. The IR divergences are logarithmically divergent if $H$ is constant.

\section{II.4. In-in Calculation of Loop Corrections}

In Sec. II.3, we have obtained the interactions and discussed the method to estimate the order of loop diagrams. In this section, we use the "in-in" formalism to perform the actual calculation of the loop corrections to the correlation function via evaluating the leading order loop diagrams. As is well known, in cosmology there is no easily defined "out" state, so "in-in" formalism is applied in order to calculate the observables, e.g. the correlation functions.

The "in-in" formalism $[34,35]$ is similar to the real-time formalism in thermal field theory. It makes use of the interaction representation of quantum field theory. In this formalism, the vacuum expectation value of an operator $\mathcal{O}$ is written as

$$
\langle\Omega|\mathcal{O}(t)| \Omega\rangle=\left\langle 0\left|\bar{T} e^{i \int_{-\infty}^{t} H_{I}\left(t^{\prime}\right) d t^{\prime}} \mathcal{O}_{I}(t) T e^{-i \int_{-\infty}^{t} H_{I}\left(t^{\prime}\right) d t^{\prime}}\right| 0\right\rangle
$$

\footnotetext{
${ }^{3}$ In fact, because of the derivative terms in the vertex factors, there are no IR divergences in Eq.(37).
} 
where $|\Omega\rangle$ is the vacuum of the interacting theory and $|0\rangle$ is the free field vacuum. The right hand side of the equation is calculated in the interaction picture. $T$ and $\bar{T}$ denote time-ordered and anti-time-ordered products.

We are considering the two point function of the curvature perturbation $\zeta$. There are two kinds of quantum corrections contributing to this Green's function, one of which is from curvature loops and the other from graviton loops. The quantum correction from eq (34) is denoted as $G_{p}^{I}(\eta)$, and the one from eq. (35) is denoted as $G_{p}^{I I}(\eta)$. Using the Feynman rules discussed in the previous subsection they are given by

$$
G_{p}^{I I}(\eta)=2 \epsilon \int_{-\infty}^{\eta} d \eta_{1} a^{2}\left(\eta_{1}\right) \mathbf{I m}\left[\zeta_{p}^{2}(\eta) \zeta_{p}^{* 2}\left(\eta_{1}\right)\right] \int \frac{d^{3} q}{(2 \pi)^{3}} 2 p^{2} \sin ^{2} \theta\left|h_{q}\left(\eta_{1}\right)\right|^{2}
$$

and

$$
G_{p}^{I I}(\eta)=-\frac{H^{2}}{4 \epsilon p^{3}} \frac{8}{16 \pi^{2}} \int_{\Lambda_{I R}} \frac{\mathrm{d} q}{q} H_{q}^{2}
$$

where $\theta$ is taken to be the angle between $p$ and $q$.

If $H_{k}$ is not $k$ dependent, the final result will be logarithmically divergent. However, in inflationary cosmology we must take into account the small $k$ dependence and study how this will impact on the physics. Focusing first on $G_{p}^{I I}$ we find

$$
\begin{aligned}
G_{p}^{I I}(\eta) & =\frac{H^{2}}{4 \epsilon p^{3}} \frac{8}{16 \pi^{2}} \int_{\Lambda_{I R}} \frac{\mathrm{d} q}{q} H_{\text {initial }}^{2}\left(\frac{q}{k_{\text {initial }}}\right)^{n_{t}} \\
& \simeq \frac{H^{2}}{4 \epsilon p^{3}} \frac{1}{16 \pi^{2}} \frac{8 H_{\text {initial }}^{2}}{n_{t}}\left(\frac{\Lambda_{I R}}{k_{\text {initial }}}\right)^{n_{t}} \\
& =-\frac{H^{2}}{4 \epsilon p^{3}} \frac{1}{16 \pi^{2}} \frac{4 H_{\text {initial }}^{2}}{\epsilon}
\end{aligned}
$$

where in the final step we have identified the IR cutoff length scale with the value of the Hubble radius at the beginning of the inflationary phase. Taking the IR cutoff momentum to be smaller would make our result probe the state of modes which are super-Hubble throughout the cosmological evolution. We have also made use of the relation (15) between $n_{t}$ and $\epsilon$.

With the same logic and method, the term $G_{p}^{I}(\eta)$ can be calculated as follows:

$$
\begin{aligned}
G_{p}^{I}(\eta)= & -16 \epsilon^{2} \mathbf{R e} \int_{-\infty}^{\eta} d \eta_{1} a^{2}\left(\eta_{1}\right) \int_{-\infty}^{\eta} d \eta_{2} a^{2}\left(\eta_{2}\right) \int d^{3} q p^{4} \sin ^{4} \theta \\
& \times\left[\theta\left(\eta_{1}-\eta_{2}\right) \zeta_{p}^{2}(\eta) \zeta_{p}^{*}\left(\eta_{1}\right) \zeta_{p}^{*}\left(\eta_{2}\right) \zeta_{p^{\prime}}\left(\eta_{1}\right) \zeta_{p^{\prime}}^{*}\left(\eta_{2}\right) h_{q}\left(\eta_{1}\right) h_{q}^{*}\left(\eta_{2}\right)\right. \\
& \left.-\frac{1}{2}\left|\zeta_{p}(\eta)\right|^{2} \zeta_{p}\left(\eta_{1}\right) \zeta_{p}^{*}\left(\eta_{2}\right) \zeta_{p^{\prime}}\left(\eta_{1}\right) \zeta_{p^{\prime}}^{*}\left(\eta_{2}\right) h_{q}\left(\eta_{1}\right) h_{q}^{*}\left(\eta_{2}\right)\right],
\end{aligned}
$$


which leads to

$$
\begin{aligned}
G_{p}^{I}(\eta) & \simeq \frac{H^{2}}{4 \epsilon p^{3}} \frac{1}{16 \pi^{2}}\left(-\frac{4 H_{\text {initial }}^{2}}{n_{t}}-\frac{4 H_{\text {initial }}^{2}}{n_{s}-1}\right) \\
& =\frac{H^{2}}{4 \epsilon p^{3}} \frac{1}{16 \pi^{2}}\left(\frac{2 H_{\text {initial }}^{2}}{\epsilon}+\frac{2 H_{\text {initial }}^{2}}{3 \epsilon-\eta}\right)
\end{aligned}
$$

The one loop corrections from $S_{4}$ do not have any IR divergences, because the terms associated with loops are always have a derivative acting on them. Hence, there are no integrals such as $\int \mathrm{d} q^{3} / q^{3}$ which result, and hence there is no IR divergence.

Notice that $G_{p}^{I}$ and $G_{p}^{I I}$ have different signs, and thus there is a possibility that the terms might cancel. In fact, if we consider a theory in which $\epsilon$ is very slowly varying on a time scale of $H^{-1}$ compared to its actual value, which often happens in models which admit eternal inflation or in which inflation simply lasts a lot longer than the minimal 60 e-foldings which it needs to last in order to be successful at resolving the flatness and horizon problems of Standard Cosmology, then

$$
\eta \simeq 2 \epsilon
$$

and the leading order IR divergences is cancelled. On the other hand, if $\frac{\dot{\epsilon}}{H \epsilon}$ is of the same order as $\epsilon$, then the analysis is more complicated, and we need to take into account the detailed time dependence of $\epsilon$.

In models in which inflation only lasts 60 e-folding the leading IR divergences do not cancel. However, the magnitude of the loop corrections is small because the phase space of IR modes is small.

\section{ENTROPY MODES}

The restriction to single field models of inflation is not very physical. We know that matter in the real world is described by a large number of fields. The inflaton is one additional field which must be added to the theory. The inflaton must also couple to other fields in order to provide a successful exit from the inflationary phase. The presence of other matter fields leads to the presence of entropy modes. Thus, when computing quantum effects on cosmological observables it is essential to take into account the effects of loops involving entropy modes.

Whereas is some circumstances adiabatic loops lead to effects which can be gauged away (as we have seen in the previous section), effects of entropy loops cannot (see e.g. [14]). Thus, if the cosmological fluctuations can be influenced by IR effects from entropy loops, these effects cannot be gauged away. They will contribute to the effective field theory of inflationary perturbations and 
can hence influence cosmological observations. In this section, we will study the IR divergences to curvature perturbation via entropy loops. We will see that these effects can be very important and that these loops effects can lead to a breakdown of perturbation theory if the self-coupling constant of the entropy modes is larger than some critical value which is much smaller than 1 . The magnitude of the infrared effects of entropy modes depends on the mass of the entropy modes and on the initial conditions. We separate this section into three parts, one of which deals with massless entropy modes, and the second subsection considers entropy modes with a small mass. These two cases will give different IR divergences. And in the final part we will deal with a more general case in which we take the Higgs field to be the inflaton as a example.

Several groups have studied entropy loops $[3,11]$ by adding a (approximately) massless scalar field $\sigma$ to the matter sector which minimally couples to gravity. The Lagrangian for $\sigma$ is

$$
\mathcal{L}=-\frac{1}{2} \partial_{\mu} \sigma \partial^{\mu} \sigma
$$

The correlation function $\langle\zeta \zeta\rangle$ does not pick up important IR contributions from the kinetic term, since the derivatives will introduce an extra power of momentum into the loop integrals which make the integrals more UV rather than IR divergent. Considering the simplest interaction between the inflaton and the entropy field,

$$
\mathcal{L}=-\frac{1}{2} \partial_{\mu} \sigma \partial^{\mu} \sigma-g \phi^{2} \sigma^{2}
$$

it appears that there are sizeable IR divergences. However, unless $g$ is very small, the entropy field $\sigma$ will acquire a large mass from the expectation value of the background inflaton field. The effective IR cutoff for entropy loops is thus the larger of either the Hubble scale (the $\sigma$ mass induced by gravitational effects) or the induced mass of the entropy mode. Thus, the IR contribution of entropy modes is tiny.

However, the conclusion that the entropy IR modes contribute little to the two point correlation function of $\zeta$ has an important loophole, which relies on the fact that the curvature perturbation is no longer constant outside Hubble radius. It is well-known that entropy fluctuations source a growing curvature perturbation. Also, the entropy self-interaction term will contribute to the curvature perturbations via the gravitational coupling. In order to study the effects of entropy perturbations, there are two ways to proceed. The first is to solve for the time dependent curvature perturbations from the equation of motion for $\zeta$ in the presence of entropy sources and then to perform the loop calculation using the in-in formalism. The second approach (the one we now adopt), is to solve the free equation of motion for $\zeta$, thus obtaining a time-independent solution, 
and then treat the source term as an interaction term. We then use the in-in formalism to obtain the corrections from IR entropy modes ${ }^{4}$.

\section{III.1. Massless Entropy Modes}

It is a general result that there is mixing between isocurvature and curvature modes. One simple example in which it is easy to see this mixing is to consider a model with a complex scalar field with a "Mexican hat" potential. The adiabatic mode corresponds to the scalar field moving in angular direction in the vacuum manifold, and the isocurvature direction is along the radius. The action describing the system is as follows [38]

$$
S=\int \mathrm{d} \eta \mathrm{d}^{3} x \frac{1}{2}\left(a^{2} \theta^{\prime 2}(R+\sigma)^{2}-a^{4} \lambda \sigma^{4}\right),
$$

where $R$ represents the radius of the circle in field space, and $\theta$ is the angle of the scalar field configuration. The field $\theta$ represents the curvature perturbation. The self interaction of the entropy modes can have various form; here we use a $\lambda \sigma^{4}$ interaction without loss of generality. If the interaction does not contain more than two time-like or spacial derivatives, then entropy loops lead to a IR divergence.

The mixing term is treated as an interaction. As we argued in Section 2, it is convenient to work in the gauge given by $\delta \theta=0$. To obtain the interaction Hamiltonian in this gauge, we start with the perturbative expansion of (48) and then (valid to first order approximation) transform the interaction term to the comoving gauge by the transformation

$$
R \delta \theta \rightarrow \zeta(-\dot{\phi} / H)
$$

The interaction Hamiltonian then becomes ${ }^{5}$

$$
\mathcal{H}_{I}=-2 a^{2} \theta_{0}^{\prime} \delta \theta^{\prime} R \sigma \rightarrow 4 a^{3} \frac{\epsilon H}{R} \zeta^{\prime} \sigma
$$

where the second arrow indicates the result after performing the gauge transformation. If the entropy modes are massless, we have the exact solution of the free entropy modes which start on sub-Hubble scales in their vacuum state:

$$
\sigma=\frac{H_{*}}{\sqrt{2 k^{3}}}(1+i k \eta) e^{-i k \eta}
$$

\footnotetext{
4 The corresponding result is equivalent to the leading order in expansion with respect to the coupling between entropic and adiabatic modes of the result in the first approach. This perturbative approach is employed in [36] in evaluating the cross-correlation between adiabatic and entropic mode and in [37] and [38] in evaluating the transfer of non-Gaussianities from the entropic to the adiabatic sector.

${ }^{5}$ In multi-field models, in general there are two types of couplings between adiabatic and entropic modes (see e.g. [39]): $\sim \xi \zeta^{\prime} \sigma$ and $\sim \mathcal{H} \xi \zeta \sigma$ with the common coefficient $\xi$ denoting the tuning rate in field space (as $\dot{\theta}$ in eq.(48)). In this work, in order to see the qualitative effect of entropic mode, we only take into account the contribution from the first type.
} 
Note that these mode functions freeze out and become constant in time on super-Hubble scales.

First, the time dependent part of tree level contribution to the two point function ${ }^{6}$ is captured by the two point mixing interaction term (50) and yields

$$
\begin{aligned}
G_{\text {tree }}= & -2 \mathbb{R e}\left\langle\int H_{I} \int H_{I} \zeta \zeta\right\rangle+\left\langle\int H_{I} \zeta \zeta \int H_{I}\right\rangle \\
= & (2 \pi)^{3} \delta^{3}\left(\mathbf{k}-\mathbf{k}^{\prime}\right)\left(\frac{4 \epsilon H}{R}\right)^{2} \\
& {\left[-4 \mathbb{R e} \int_{-\infty}^{\eta} \mathrm{d} \eta_{2} a^{3}\left(\eta_{2}\right) \int_{-\infty}^{\eta_{2}} \mathrm{~d} \eta_{1} a^{3}\left(\eta_{1}\right) \sigma_{k}\left(\eta_{1}\right) \zeta_{k}^{\prime}\left(\eta_{1}\right) \sigma_{k}^{*}\left(\eta_{2}\right) \zeta_{k}^{\prime}\left(\eta_{2}\right) \zeta_{k}^{*}(\eta) \zeta_{k}^{*}(\eta)\right.} \\
& \left.+2 \int_{-\infty}^{\eta} \mathrm{d} \eta_{1} a^{3}\left(\eta_{1}\right) \int_{-\infty}^{\eta} \mathrm{d} \eta_{2} a^{3}\left(\eta_{2}\right) \sigma_{k}\left(\eta_{1}\right) \zeta_{k}^{\prime}\left(\eta_{1}\right) \zeta_{k}^{*}(\eta) \zeta_{k}(\eta) \sigma_{k}^{*}\left(\eta_{2}\right) \zeta_{k}^{\prime *}\left(\eta_{2}\right)\right] \\
= & (2 \pi)^{3} \delta^{3}\left(\mathbf{k}-\mathbf{k}^{\prime}\right) \frac{8 \epsilon M_{p l}^{2}}{R^{2}} \frac{H^{2}}{4 \epsilon k^{3}} \times[\log (-k \eta)]^{2}
\end{aligned}
$$

The tree level correction from the entropy modes is given by the following diagram and yields

$$
\frac{\frac{\epsilon H}{R H^{3}}}{H^{2} / \epsilon}-\frac{\frac{\epsilon H}{R H^{3}}}{H^{2}} \underset{H^{2} / \epsilon}{\sim} \frac{\epsilon}{R^{2}} \frac{H^{2}}{\epsilon} \times[\log (-k \eta)]^{2} .
$$

Here, the solid lines denote curvature correlations $\langle\zeta \zeta\rangle \sim \frac{H^{2}}{\epsilon}$, and the dotted lines are entropy modes with propagator $\langle\sigma \sigma\rangle \sim H^{2}$. The interaction vertex is proportional to $\frac{\epsilon H}{R}$. In this estimate, the extra $H^{3}$ in the denominators of the interaction terms arise from the $\mathrm{H}$-dependence of the scale factor $a^{3}=-\frac{1}{H^{3} \eta^{3}}$ arising in the interactions. The entropy perturbations source the curvature perturbation via

$$
\dot{\zeta} \sim \delta s
$$

and this explains why the tree level result above contains the two factors of $\log (-k \eta) \sim t$.

So far, we have neglected the mass and self-interactions of the entropy field. For the moment, we will assume that the mass of the entropy modes is small and compute the effects of the selfinteractions. To be specific, we choose a $\sigma^{4}$ interaction

$$
\mathcal{H}_{I}=a^{4} \lambda \sigma^{4}
$$

and will go on to study the resulting entropy loop correction to the curvature two point function.

\footnotetext{
${ }^{6}$ See [36] for a related calculation of the corrections to the adiabatic power spectrum due to the coupling between adiabatic and entropic modes.
} 
It is given by the following Feynman diagram

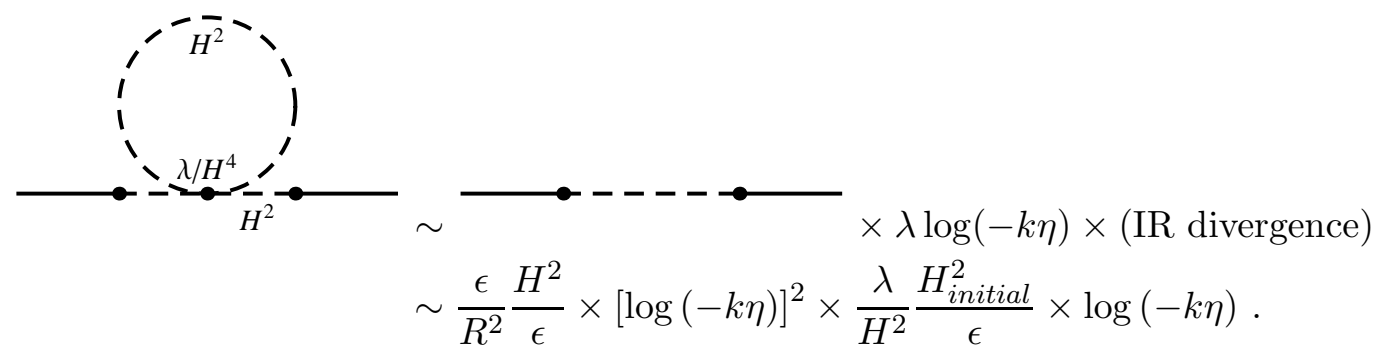

As in Section II.4 (Eq. (42)), the power-law infrared divergence leads to the factor of $H_{\text {initial }}^{2} / \epsilon$, where the IR cutoff length scale is set to be the Hubble radius at the beginning of inflation. The physical reason for having the extra term $\log (-k \eta)$ is that the entropy mode also sources itself and the effect increases with time. The IR divergence is expresed via the term $\frac{H_{\text {initial }}^{2}}{\epsilon}$ where the IR cutoff length scale is (as in Section 2) set to be the Hubble radius at the beginning of inflation. This divergence is similar in structure to what we found in Eq. 42. However, in this case there can be no cancellation in the limit of very slow-roll inflation. Note that the logarithm divergences of pure de Sitter space has changed to a power law divergence. Summarizing, the entropy loop correction to the curvature two point function is given by

$$
\begin{aligned}
G_{\text {loop }} & =2 \mathbb{I m}\left\langle\int H_{I} \int H_{I} \int H_{I} \zeta \zeta\right\rangle-2 \mathbb{I m}\left\langle\int H_{I} \int H_{I} \zeta \zeta \int H_{I}\right\rangle \\
& =(2 \pi)^{3} \delta^{3}\left(\mathbf{k}-\mathbf{k}^{\prime}\right) \lambda \frac{14 M_{p l}^{2}}{3 \pi^{2} R^{2}} \frac{H^{2}}{4 \epsilon k^{3}}\left(\frac{H_{\text {initial }}}{H}\right)^{2} \times[\log (-k \eta)]^{3} .
\end{aligned}
$$

Comparing the tree level result with the one loop contribution we see that perturbation theory breaks down when

$$
\lambda \frac{7}{12 \pi^{2}} \frac{1}{\epsilon}\left(\frac{H_{\text {initial }}}{H}\right)^{2} \times[\log (-k \eta)]>1 .
$$

Using dynamical renormalization group techniques [40], the temporal logarithm divergences $[\log (-k \eta)]$ can be resummed. In fact, one way to interpret this result is that interaction generate a mass of order $\frac{\lambda}{\epsilon}\left(\frac{H_{\text {initial }}}{H}\right)^{2}$.

After doing this, the condition for the breakdown of perturbation theory changed slightly and becomes

$$
\lambda \frac{1}{4 \pi^{2}} \frac{1}{\epsilon}\left(\frac{H_{\text {initial }}}{H}\right)^{2}>1 .
$$

which comes from the subleading term in the loop corrections and is still sensitive to the initial condition. This constraint on $\lambda$ is severe, in particular in models with a very long period of inflation when the ratio of $H$ values is large. 


\section{III.2. Massive Entropy Modes}

Massive entropy modes are not constant outside the Hubble radius (even at level of free field theory). The free field equation of motion is

$$
\sigma_{k}^{\prime \prime}-\frac{2}{\eta} \sigma_{k}^{\prime}+\left(k^{2}+\frac{m^{2}}{H^{2} \eta^{2}}\right) \sigma_{k}=0 .
$$

After choosing the Bunch-Davis vacuum at past infinity the solutions are

$$
\sigma_{k}=-i e^{i\left(\nu+\frac{1}{2}\right) \frac{\pi}{2}} \frac{\sqrt{\pi}}{2} H(-\eta)^{\frac{3}{2}} \mathrm{H}_{\nu}^{(1)}(-k \eta) \quad \text { for } \quad \frac{m^{2}}{H^{2}}<\frac{4}{9}
$$

where $H_{\nu}$ indicates a Hankel function and

$$
\nu=\sqrt{\frac{9}{4}-\frac{m^{2}}{H^{2}}} .
$$

If $-k \eta \ll 1$ (super-Hubble scales), the values of the modes are slowly decreasing in time:

$$
\sigma_{k} \simeq-e^{i\left(\nu+\frac{1}{2}\right) \frac{\pi}{2}} \frac{2^{\nu-1}}{\sqrt{\pi}} \Gamma(\nu) \frac{H}{k^{\frac{3}{2}}}(-k \eta)^{-\nu+\frac{3}{2}}
$$

In the case of $m \ll H$ we have $\nu \simeq \frac{3}{2}-\frac{1}{3} \frac{m^{2}}{H^{2}}$, and hence

$$
(-\eta)^{-\nu+\frac{3}{2}} \simeq(a(\eta) H)^{-\frac{1}{3} \frac{m^{2}}{H^{2}}} \simeq e^{-\frac{1}{3} \frac{m^{2}}{H^{2}}(H t+\ln H)}
$$

Thus the entropy modes will have decayed away on a time scale of $H t \sim 3 \frac{H^{2}}{m^{2}}$. Assuming that $m \sim 10^{-2} H$, then the decay will have occurred after $10^{4}$ e-foldings. Thus, the effective IR cutoff for the massive entropy modes is

$$
\Lambda_{I R} \sim H e^{-3 \frac{H^{2}}{m^{2}}}
$$

If inflation does not last as long as $3 \frac{H^{2}}{m^{2}}$ e-foldings, the IR cutoff is the same as for massless entropy modes when it is set by the initial value of the Hubble radius, as we have previously discussed.

We now first calculate the tree level correction to the curvature correlation function. The calculation is similar to the analysis of Eq. (52) in the case of massless entropy. We will just write down the leading order terms:

$$
\begin{aligned}
G_{\text {tree }}= & (2 \pi)^{3} \delta^{3}\left(\mathbf{k}-\mathbf{k}^{\prime}\right)\left(\frac{4 \epsilon H}{R}\right)^{2} \times\left[\frac{(-k \eta)^{-\nu+\frac{3}{2}}}{4 \epsilon^{2}\left(\nu-\frac{3}{2}\right)^{2} k^{3}}\right. \\
& \left.-\frac{4^{\nu-1}(\cos (2 \pi \nu)-\cos (4 \pi \nu)) \Gamma(\nu)^{2}}{2 \pi^{2} k^{3} \epsilon^{2}(2 \nu-1)}(-k \eta)^{-2 \nu+1}\right]
\end{aligned}
$$

The first term in the bracket is the same as Eq. (52), with the $\log ^{2}(-k \eta)$ divergence being recoved in the limit of $\nu \rightarrow \frac{3}{2}$, and the second term is zero in this limit. 
The one loop result is complicated, and we here only show the order of magnitude of the result which can be derived from the in-in formalism similar to in Eq. (57), or can be estimated as in Eq. (56). The result is

$$
G_{\text {loop }} \sim G_{\text {tree }} \times \lambda \frac{1}{\epsilon}\left(\frac{H_{\text {initial }}}{H}\right)^{2} \frac{(-k \eta)^{x\left(-\frac{3}{2}+\nu\right)}}{\nu-\frac{3}{2}}
$$

where $x$ is a non-negative integer of the order one. Comparing with the result for massless entropy modes, we see that the logarithm divergence in time becomes a power law divergence.

Comparing the magnitudes of the tree level and one loop terms again leads to a condition on $\lambda$ in order that perturbation theory remain valid. The most conservative constraint on the coupling constant $\lambda$, independent whether the entropy mode is massless or massive, is

$$
\lambda<\epsilon e^{-2 N \epsilon} \frac{1}{N^{\prime}}
$$

where $N$ is the total e-folding number of the inflationary period, and $N^{\prime}$ denotes how many efoldings before the end of inflation that the $k$ mode corresponding to the external line of the curvature perturbation exits the Hubble radius. Choosing the fiducial values $N \sim 60, \epsilon \sim 0.1$ and $N^{\prime} \sim \mathcal{O}(1 \div 10)$, then

$$
\lambda<10^{-7} \div 10^{-6}
$$

However, in large field models of inflation the value of $N$ is much larger than the minimal value chosen to obtain the above estimate, and then the constraint (68) will be much more stringent than the result (69).

\section{III.3. Two Loops and Higgs Field}

In this subsection, we will discuss a more general situation which is expected to occur during inflation. Besides the inflaton, we expect physics to contain many other scalar fields, such as the Higgs field(s), moduli fields, and superpartners of fermions. There is the possibility that these scalars do not directly interact with the inflaton but only interact with curvature perturbations via gravity. If the masses of these scalar fields are light compared to the Hubble scale (which we expect to be true in the case of the Higgs field(s)), then the modes of these scalar field will decay at $3 \frac{H^{2}}{m^{2}}$ e-foldings after Hubble radius crossing, as discussed above. For example, the decay of the

Higgs field with mass $\mathcal{O}(100) G e V$ begins at $\frac{H^{2}}{m^{2}} \sim \mathcal{O}\left(10^{25}\right)$ e-foldings after the modes leave the Hubble horizon. Therefore, for fields with a small mass, the IR cutoff will usually be given by the initial Hubble radius at the beginning of the inflationary phase. 
The interactions terms for the scalars come from self-interactions

$$
\begin{aligned}
S_{I} & =\int \mathrm{d} \eta \mathrm{d}^{3} x N \sqrt{h}\left(-\lambda \sigma^{4}\right)=\int \mathrm{d} \eta \mathrm{d}^{3} x a^{4} e^{3 \zeta+\zeta^{\prime} /(a H)}\left(-\lambda \sigma^{4}\right) \\
& \supset-\int \mathrm{d} \eta \mathrm{d}^{3} x a^{4}\left(\frac{9}{2} \zeta^{2}+\frac{3}{a H} \zeta \zeta^{\prime}+\frac{1}{2 a^{2} H^{2}} \zeta^{\prime 2}\right) \lambda \sigma^{4}
\end{aligned}
$$

Here we neglect the interactions with the graviton, and only consider the interaction with the curvature perturbation $\zeta$, since the two point correction function of curvature perturbations are considered. This kind of interaction can lead to two types of loop correction to the two point function of $\zeta$.

We can estimate the magnitude of the loop corrections by counting powers in $H$ and $\epsilon$ and making use of the power-law IR divergences which depend on the initial value of the Hubble scale, as in previous subsection. The Feynman diagram and the scaling of its contribution to the two point function is

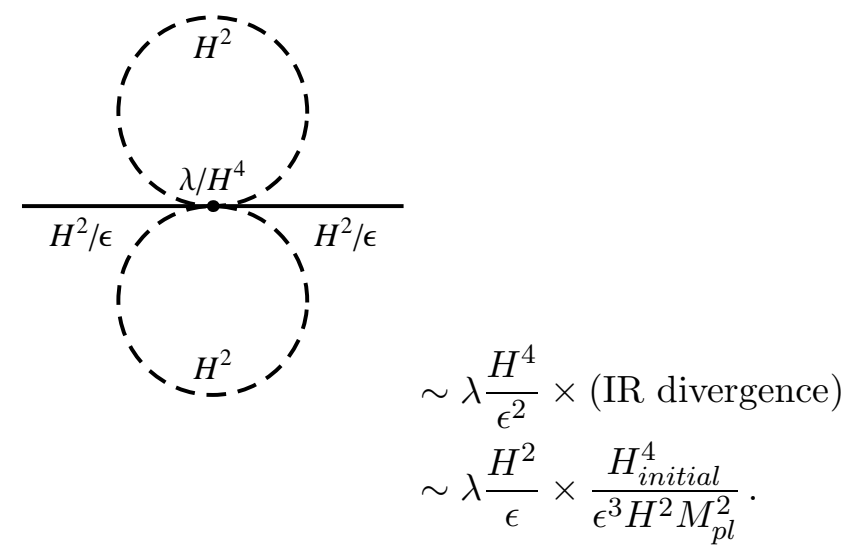

The magnitude of the loop correction can be calculated by in-in formalism and yields

$$
\begin{aligned}
G_{\text {2loop }} & =-2 \operatorname{Im}\left\langle\int H_{I} \zeta \zeta\right\rangle \\
& =(2 \pi)^{3} \delta^{3}\left(\mathbf{k}-\mathbf{k}^{\prime}\right) \frac{H^{2}}{2 \epsilon k^{3}} \frac{3}{8(2 \pi)^{4} \epsilon^{3}} \frac{H_{\text {initial }}^{2}}{H^{2}} \frac{H_{\text {initial }}^{2}}{M_{p}^{2}} \times \lambda .
\end{aligned}
$$

We can now ask what constraint on the coupling constant results. The answer depends on how long inflation lasts and how large $H$ is. If we assume that there was eternal inflation in the early universe, and that thus the density fluctuations initially were very large (on scales which are obviously completely irrelevant to current observations), i.e.

$$
\left(\frac{\delta \rho}{\rho}\right)^{2}=\frac{H_{\text {initial }}^{2}}{2 \epsilon(2 \pi)^{2} M_{p}^{2}} \geq 1,
$$

then we can derive the following constraint on the coupling constant $\lambda$ : If we require that the two loop correction is not larger than the tree level value, then

$$
\lambda<10^{-10} \times \epsilon^{2}<10^{-12},
$$


where $10^{-10}$ is from the thermal fluctuations of CMB. Thus, if we want to trust the effective field theory of eternal inflation, all scalar fields with the small mass must have a self-interaction coupling coonstant which is smaller than $10^{-12}$.

\section{DISCUSSION}

In this paper, we have computed one loop corrections to the two-point correlation function of the scalar field $\zeta$ which characterizes the curvature perturbations. We emphasized that the kdependence of the Hubble scale during inflation will change the IR logarithmic divergences of pure de Sitter space into power law divergences. The power divergence implies that the IR divergences are sensitive to the initial value of the Hubble parameter, the natural IR cutoff scale in the context of inflationary cosmology.

We argued that the best choice of the gauge to perform the computation of IR looops in single field inflation models is the comoving gauge, since in it the perturbation variable (namely $\zeta$ ) is not time-dependent on super-Hubble scales.

We showed how to estimate the IR divergences in one loop corrections to the two point correlation function of $\zeta$, and we also used the in-in formalism to calculate the one loop diagram, both of which are consistent. The correction is of the order $\mathcal{O}\left(\frac{H_{\text {initial }}^{2}}{\epsilon}\right)$. In single field models of inflation, then if the inflationary phase lasts long, the requirement that the slow roll condition on $\epsilon$ is preserved in time will lead to some cancellations of the loop corrections, and the semi-classical calculation breaks down only when $H_{\text {initial }}^{2} \sim 1$. The cancellation of the leading IR divergences in single field inflation models is consistent with the semi-classical relation obtained in [9].

However, in multiple field inflation models, the curvature perturbation $\zeta$ is not conserved on super-Hubble scales. The entropy modes source the curvature perturbation and also self-interact. Neither the kinetic term of the entropy field nor the interaction terms coupling the entropy field to the inflaton lead to an IR divergence, but the self-interaction terms do lead to IR divergences. Massless entropy modes will lead to logarithm divergences in conformal time, while the massive (but low mass) ones will lead to power law divergences in time. Estimating the ratio of the one loop to tree level results leads to constraints on the self-coupling of the entropy modes which have to be satisfied in order to avoid the breakdown of perturbation theory. In the two fields inflation models, for a $\lambda \sigma^{4}$ interaction term (where $\sigma$ is the entropy field), the constraint takes the form $\lambda<10^{-7} \div 10^{-6}$ if we require that the effective field theory for inflation remain valid for more than 60 e-folding. Because this constraint is on the self-coupling, we believe that it is rather 
generic. If inflation lasts longer than the minimal duration it must last in order to address the cosmological problems of the Standard Big Bang model, then the constraints on $\lambda$ become much more restrictive. In more general cases, any scalar (for example, the Higgs field) which does not couple directly to the inflaton field, couples to gravity. The leading order correction which such a field has on the curvature perturbation is from a two-loop diagram, and the requirement that the two-loop correction is not larger than the tree level result leads to a constraint of the self-coupling of the scalar field of the form $\lambda<10^{-12}$ if the inflaton field starts in the region of eternal inflation.

Our analysis has implications for the recent debate about the stability of de Sitter space. There is one camp [41] arguing for the stability of de Sitter space even in the presence of fluctuations based on performing the analysis in Euclidean de Sitter space. A second camp [42] argues that in a cosmological context a de Sitter phase is unstable because of large IR back-reaction effects. The renormalization prescription breaks the de Sitter invariance as can be seen either by imposing an initial space-like Cauchy surface (e.g. the initial time of the inflationary phase), or by setting the IR cutoff to be fixed in comoving coordinates (see e.g. [43]) for a recent comparison of these two camps). We have shown that in the presence of entropy fluctuations, the effects which lead the second camp to argue that de Sitter space is unstable will be much larger in magnitude. Thus, our analysis may imply that entropy modes lead to an enhanced decay rate of de Sitter space.

\section{ACKNOWLEDGEMENTS}

We would like to thank L. Leblond, E. Lim, S. Shandera and R. Woodard for interesting conversations. X. G. is supported by ANR (Agence Nationale de la Recherche) grant "STRCOSMO" ANR-09-BLAN-0157-01. W. X. is supported in part by a Schulich Fellowship , and R.B. is supported in part by an NSERC Discovery grant and by funds from the Canada Research Chair program. R.B. wishes to thank Patrick Peter and Jerome Martin for an invitation to the IAP during which this project was finalized. This visit was supported by the grant "PICS Cosmologie de l'Univers Primordial" to the IAP. We wish to thank M. Sloth, A. Riotto, R. Woodard, L. Leblond and M. Zaldarriaga for comments on the manuscript.

[1] V. F. Mukhanov, H. A. Feldman and R. H. Brandenberger, "Theory of cosmological perturbations. Part 1. Classical perturbations. Part 2. Quantum theory of perturbations. Part 3. Extensions," Phys. Rept. 215, 203 (1992). 
[2] N. Bartolo, S. Matarrese, A. Riotto, "Nongaussianity from inflation," Phys. Rev. D65, 103505 (2002). [hep-ph/0112261];

N. Bartolo, E. Komatsu, S. Matarrese et al., "Non-Gaussianity from inflation: Theory and observations," Phys. Rept. 402, 103-266 (2004). [astro-ph/0406398];

X. Chen, "Primordial Non-Gaussianities from Inflation Models," Adv. Astron. 2010, 638979 (2010) [arXiv:1002.1416 [astro-ph.CO]].

[3] S. Weinberg, "Quantum contributions to cosmological correlations," Phys. Rev. D 72, 043514 (2005) [arXiv:hep-th/0506236].

[4] N. C. Tsamis and R. P. Woodard, "Relaxing The Cosmological Constant," Phys. Lett. B 301, 351 (1993);

N. C. Tsamis and R. P. Woodard, "The Physical basis for infrared divergences in inflationary quantum gravity," Class. Quant. Grav. 11, 2969 (1994);

N. C. Tsamis and R. P. Woodard, "Strong infrared effects in quantum gravity," Annals Phys. 238, 1 (1995);

N. C. Tsamis and R. P. Woodard, "The quantum gravitational back-reaction on inflation," Annals Phys. 253, 1 (1997) [arXiv:hep-ph/9602316];

N. C. Tsamis and R. P. Woodard, "Quantum Gravity Slows Inflation," Nucl. Phys. B 474, 235 (1996) [arXiv:hep-ph/9602315];

N. C. Tsamis and R. P. Woodard, "One Loop Graviton Self-Energy In A Locally De Sitter Background," Phys. Rev. D 54, 2621 (1996) [arXiv:hep-ph/9602317].

[5] L. R. W. Abramo, R. H. Brandenberger and V. F. Mukhanov, "The energy-momentum tensor for cosmological perturbations," Phys. Rev. D 56, 3248 (1997) [arXiv:gr-qc/9704037];

V. F. Mukhanov, L. R. W. Abramo and R. H. Brandenberger, "On the back reaction problem for gravitational perturbations," Phys. Rev. Lett. 78, 1624 (1997) [arXiv:gr-qc/9609026];

L. R. W. Abramo and R. P. Woodard, "One loop back reaction on chaotic inflation," Phys. Rev. D 60, 044010 (1999) [arXiv:astro-ph/9811430].

[6] N. D. Birrell and P. C. W. Davies, "Quantum Fields In Curved Space," Cambridge, Uk: Univ. Pr. ( 1982) $340 p$.

[7] L. Senatore, M. Zaldarriaga, "On Loops in Inflation," JHEP 1012, 008 (2010). [arXiv:0912.2734 [hepth]].

[8] W. Xue, K. Dasgupta and R. Brandenberger, "Cosmological UV/IR Divergences and de-Sitter Spacetime," Phys. Rev. D 83, 083520 (2011) [arXiv:1103.0285 [hep-th]].

[9] S. B. Giddings, M. S. Sloth, "Semiclassical relations and IR effects in de Sitter and slow-roll spacetimes," JCAP 1101, 023 (2011). [arXiv:1005.1056 [hep-th]].

[10] S. B. Giddings and M. S. Sloth, "Cosmological observables, IR growth of fluctuations, and scaledependent anisotropies," Phys. Rev. D 84, 063528 (2011) [arXiv:1104.0002 [hep-th]]. 
[11] D. Seery, "Infrared effects in inflationary correlation functions," Class. Quant. Grav. 27, 124005 (2010). [arXiv:1005.1649 [astro-ph.CO]].

[12] M. S. Sloth, "On the one loop corrections to inflation and the CMB anisotropies," Nucl. Phys. B748, 149-169 (2006). [astro-ph/0604488];

M. S. Sloth, "On the one loop corrections to inflation. II. The Consistency relation," Nucl. Phys. B775, 78-94 (2007). [hep-th/0612138];

D. Seery, "One-loop corrections to a scalar field during inflation," JCAP 0711, 025 (2007) [arXiv:0707.3377 [astro-ph]];

D. Seery, "One-loop corrections to the curvature perturbation from inflation," JCAP 0802, 006 (2008). [arXiv:0707.3378 [astro-ph]]. N. Bartolo, A. Riotto, "Possibly Large Corrections to the Inflationary Observables," Mod. Phys. Lett. A23, 857-862 (2008) [arXiv:0711.4003 [astro-ph]];

N. Bartolo, S. Matarrese, M. Pietroni et al., "On the Physical Significance of Infra-red Corrections to Inflationary Observables," JCAP 0801, 015 (2008) [arXiv:0711.4263 [astro-ph]];

Y. Urakawa, K. -i. Maeda, "One-loop Corrections to Scalar and Tensor Perturbations during Inflation in Stochastic Gravity,” Phys. Rev. D78, 064004 (2008). [arXiv:0801.0126 [hep-th]];

K. Enqvist, S. Nurmi, D. Podolsky et al., "On the divergences of inflationary superhorizon perturbations," JCAP 0804, 025 (2008). [arXiv:0802.0395 [astro-ph]];

E. Dimastrogiovanni, N. Bartolo, "One-loop graviton corrections to the curvature perturbation from inflation," JCAP 0811, 016 (2008); [arXiv:0807.2790 [astro-ph]];

X. Gao, F. Xu, "Loop Corrections to Cosmological Perturbations in Multi-field Inflationary Models," JCAP 0907, 042 (2009). [arXiv:0905.0405 [hep-th]];

D. Campo, "Quantum corrections during inflation and conservation of adiabatic perturbations," Phys. Rev. D81, 043535 (2010). [arXiv:0908.3642 [hep-th]];

J. Kumar, L. Leblond, A. Rajaraman, "Scale Dependent Local Non-Gaussianity from Loops," JCAP 1004, 024 (2010). [arXiv:0909.2040 [astro-ph.CO]]. N. Bartolo, E. Dimastrogiovanni, A. Vallinotto, "One-loop corrections to the power spectrum in general single-field inflation," JCAP 1011, 003 (2010). [arXiv:1006.0196 [astro-ph.CO]];

C. T. Byrnes, M. Gerstenlauer, A. Hebecker, S. Nurmi and G. Tasinato, "Inflationary Infrared Divergences: Geometry of the Reheating Surface vs. delta N Formalism," JCAP 1008, 006 (2010) [arXiv:1005.3307 [hep-th]].

[13] M. Gerstenlauer, A. Hebecker and G. Tasinato, "Inflationary Correlation Functions without Infrared Divergences," arXiv:1102.0560 [astro-ph.CO];

Y. Urakawa, T. Tanaka, "IR divergence does not affect the gauge-invariant curvature perturbation," Phys. Rev. D82, 121301 (2010). [arXiv:1007.0468 [hep-th]];

D. Chialva and A. Mazumdar, "Eliminating infrared divergences in an inflationary cosmology," arXiv:1103.1312 [hep-th];

A. Riotto and M. S. Sloth, "The probability equation for the cosmological comoving curvature pertur- 
bation," JCAP 1110, 003 (2011) [arXiv:1103.5876 [astro-ph.CO]].

[14] G. Geshnizjani and R. Brandenberger, "Back reaction and local cosmological expansion rate," Phys. Rev. D 66, 123507 (2002) [arXiv:gr-qc/0204074].

[15] C. P. Burgess, L. Leblond, R. Holman et al., "Super-Hubble de Sitter Fluctuations and the Dynamical RG," JCAP 1003, 033 (2010). [arXiv:0912.1608 [hep-th]];

C. P. Burgess, R. Holman, L. Leblond et al., "Breakdown of Semiclassical Methods in de Sitter Space," JCAP 1010, 017 (2010). [arXiv:1005.3551 [hep-th]].

[16] A. Riotto, M. S. Sloth, "On Resumming Inflationary Perturbations beyond One-loop," JCAP 0804, 030 (2008). [arXiv:0801.1845 [hep-ph]];

B. Garbrecht and G. Rigopoulos, "Self Regulation of Infrared Correlations for Massless Scalar Fields during Inflation," Phys. Rev. D 84, 063516 (2011) [arXiv:1105.0418 [hep-th]].

[17] A. D. Linde, "Infrared Problem in Thermodynamics of the Yang-Mills Gas," Phys. Lett. B 96, 289 (1980).

[18] B. Losic and W. G. Unruh, "Cosmological Perturbation Theory in Slow-Roll Spacetimes," Phys. Rev. Lett. 101, 111101 (2008) [arXiv:0804.4296 [gr-qc]];

W. G. Unruh and B. Losic, "Aspects of nonlinear perturbations in cosmological models," Class. Quant. Grav. 25, 154012 (2008).

[19] J. M. Bardeen, "Gauge Invariant Cosmological Perturbations," Phys. Rev. D22, 1882-1905 (1980).

[20] W. Unruh, "Cosmological long wavelength perturbations," astro-ph/9802323.

[21] L. R. Abramo and R. P. Woodard, "No one loop back-reaction in chaotic inflation," Phys. Rev. D 65, 063515 (2002) [arXiv:astro-ph/0109272].

[22] G. Geshnizjani and R. Brandenberger, "Back reaction of perturbations in two scalar field inflationary models," JCAP 0504, 006 (2005) [arXiv:hep-th/0310265].

[23] Y. Urakawa, T. Tanaka, "IR divergence does not affect the gauge-invariant curvature perturbation," Phys. Rev. D82, 121301 (2010). [arXiv:1007.0468 [hep-th]];

Y. Urakawa, T. Tanaka, "Influence on Observation from IR Divergence during Inflation. I.," Prog. Theor. Phys. 122, 779-803 (2009). [arXiv:0902.3209 [hep-th]].

[24] R. H. Brandenberger and C. Vafa, "Superstrings in the Early Universe," Nucl. Phys. B 316, 391 (1989).

[25] R. H. Brandenberger, "String Gas Cosmology," arXiv:0808.0746 [hep-th].

[26] R. H. Brandenberger, A. Nayeri, S. P. Patil and C. Vafa, "Tensor Modes from a Primordial Hagedorn Phase of String Cosmology," Phys. Rev. Lett. 98, 231302 (2007) [hep-th/0604126].

[27] C. Cheung, P. Creminelli, A. L. Fitzpatrick, J. Kaplan and L. Senatore, "The Effective Field Theory of Inflation," JHEP 0803, 014 (2008) [arXiv:0709.0293 [hep-th]].

[28] S. Weinberg, "Ultraviolet Divergences in Cosmological Correlations," Phys. Rev. D 83, 063508 (2011) [arXiv:1011.1630 [hep-th]].

[29] M. Sasaki, "Large Scale Quantum Fluctuations in the Inflationary Universe," Prog. Theor. Phys. 76, 1036 (1986). 
[30] V. F. Mukhanov, "Gravitational Instability Of The Universe Filled With A Scalar Field," JETP Lett. 41, 493 (1985) [Pisma Zh. Eksp. Teor. Fiz. 41, 402 (1985)].

[31] J. M. Maldacena, "Non-Gaussian features of primordial fluctuations in single field inflationary models," JHEP 0305, 013 (2003) [astro-ph/0210603].

[32] D. Seery, J. E. Lidsey and M. S. Sloth, "The inflationary trispectrum," JCAP 0701, 027 (2007) [astro$\mathrm{ph} / 0610210]$.

[33] P. R. Jarnhus and M. S. Sloth, "de Sitter limit of inflation and nonlinear perturbation theory," JCAP 0802, 013 (2008) [arXiv:0709.2708 [hep-th]].

[34] J. Schwinger, Proc. Nat. Acad. Sci. US 46, 1401 (1961);

K. T. Mahanthappa, "Multiple production of photons in quantum electrodynamics," Phys. Rev. 126, 329 (1962);

P. M. Bakshi and K. T. Mahanthappa, "Expectation value formalism in quantum field theory. 1," J. Math. Phys. 4, 1 (1963);

P. M. Bakshi and K. T. Mahanthappa, "Expectation value formalism in quantum field theory. 2," J. Math. Phys. 4, 12 (1963).

[35] L. Keldysh, Soviet Physics JETP 20, 1018 (1965).

[36] X. Gao, "On Cross-correlations between Curvature and Isocurvature Perturbations during Inflation," JCAP 1002, 019 (2010) [arXiv:0908.4035 [hep-th]].

[37] X. Chen and Y. Wang, "Large non-Gaussianities with Intermediate Shapes from Quasi-Single Field Inflation," Phys. Rev. D 81, 063511 (2010) [arXiv:0909.0496 [astro-ph.CO]].

[38] X. Chen and Y. Wang, "Quasi-Single Field Inflation and Non-Gaussianities," JCAP 1004, 027 (2010) [arXiv:0911.3380 [hep-th]].

[39] D. Langlois and S. Renaux-Petel, "Perturbations in generalized multi-field inflation," JCAP 0804, 017 (2008) [arXiv:0801.1085 [hep-th]].

[40] C. P. Burgess, L. Leblond, R. Holman et al., "Super-Hubble de Sitter Fluctuations and the Dynamical RG," JCAP 1003, 033 (2010). [arXiv:0912.1608 [hep-th]].

[41] A. Rajaraman, J. Kumar, L. Leblond, "Constructing Infrared Finite Propagators in Inflating Spacetime," Phys. Rev. D82, 023525 (2010). [arXiv:1002.4214 [hep-th]];

D. Marolf and I. A. Morrison, "The IR stability of de Sitter: Loop corrections to scalar propagators," Phys. Rev. D 82, 105032 (2010) [arXiv:1006.0035 [gr-qc]];

A. Rajaraman, "On the proper treatment of massless fields in Euclidean de Sitter space," Phys. Rev. D82, 123522 (2010). [arXiv:1008.1271 [hep-th]];

D. Marolf and I. A. Morrison, "The IR stability of de Sitter QFT: results at all orders," arXiv:1010.5327 [gr-qc];

A. Higuchi, D. Marolf and I. A. Morrison, "On the Equivalence between Euclidean and In-In Formalisms in de Sitter QFT," arXiv:1012.3415 [gr-qc]. 
[42] L. H. Ford, "Quantum Instability Of De Sitter Space-Time," Phys. Rev. D 31, 710 (1985);

E. Mottola, "Particle Creation In De Sitter Space," Phys. Rev. D 31, 754 (1985);

P. Mazur and E. Mottola, "Spontaneous Breaking Of De Sitter Symmetry By Radiative Effects," Nucl. Phys. B 278, 694 (1986);

J. H. Traschen and C. T. Hill, "Instability Of De Sitter Space On Short Time Scales," Phys. Rev. D 33, 3519 (1986);

A. M. Polyakov, "De Sitter Space and Eternity," Nucl. Phys. B 797, 199 (2008) [arXiv:0709.2899 [hepth]];

E. T. Akhmedov, P. V. Buividovich and D. A. Singleton, "De Sitter space and perpetuum mobile," arXiv:0905.2742 [gr-qc];

A. M. Polyakov, "Decay of Vacuum Energy," Nucl. Phys. B 834, 316 (2010) [arXiv:0912.5503 [hep-th]];

D. Krotov and A. M. Polyakov, "Infrared Sensitivity of Unstable Vacua," arXiv:1012.2107 [hep-th];

E. T. Akhmedov, "IR divergences and kinetic equation in de Sitter space. Poincare patch: Principal series," arXiv:1110.2257 [hep-th].

[43] K. Larjo and D. A. Lowe, "Initial states and infrared physics in locally de Sitter spacetime," arXiv:1112.5425 [hep-th]. 